International Journal of Modern Physics A

(C) World Scientific Publishing Company

\title{
Testing Higgs Coupling Precision and New Physics Scales at Lepton Colliders
}

\author{
Shao-Feng Ge * \\ Max-Planck-Institut für Kernphysik, Heidelberg, Germany \\ gesf02@gmail.com \\ Hong-Jian $\mathrm{He}$ \\ Institute of Modern Physics and Center for High Energy Physics, \\ Tsinghua University, Beijing 100084, China \\ Center for High Energy Physics, Peking University, Beijing 100871, China \\ hjhe@tsinghua.edu.cn \\ Rui-Qing Xiao \\ Institute of Modern Physics and Center for High Energy Physics, \\ Tsinghua University, Beijing 100084, China \\ Lawrence Berkeley National Laboratory, Berkeley, California 94720, USA \\ ruiqingxiao@lbl.gov
}

Received Day Month Year

Revised November 21, 2018

\begin{abstract}
The next-generation lepton colliders, such as CEPC, FCC-ee, and ILC will make precision measurement of the Higgs boson properties. We first extract the Higgs coupling precision from Higgs observables at CEPC to illustrate the potential of future lepton colliders. Depending on the related event rates, the precision can reach percentage level for most couplings. Then, we try to estimate the new physics scales that can be indirectly probed with Higgs and electroweak precision observables. The Higgs observables, together with the existing electroweak precision observables, can probe new physics up to $10 \mathrm{TeV}\left(40 \mathrm{TeV}\right.$ for the gluon-related operator $\mathcal{O}_{g}$ ) at $95 \%$ C.L. Including the $Z / W$ mass measurements and $Z$-pole observables at CEPC further pushes the limit up to $35 \mathrm{TeV}$. Although $Z$-pole running is originally for the purpose of machine calibration, it can be as important as the Higgs observables for probing the new physics scales indirectly. The indirect probe of new physics scales at lepton colliders can mainly cover the energy range to be explored by the following hadron colliders of $p p(50-100 \mathrm{TeV})$, such as SPPC and FCC-hh.
\end{abstract}

Keywords: Lepton Collider; Dimension-6 Operator; Collider Phenomenology.

PACS numbers:

*Presenter of the talk "New Physics Scales to be Probed at Lepton Colliders" at the IAS Program on High Energy Physics on January 11, 2016. 


\section{Introduction}

With the discovery of Higgs boson ${ }^{1}$ at LHC, ${ }^{2}$ the spectrum of the SM has been completed. This culminates in the success of searches that lasted for decades. ${ }^{3}$ Nevertheless, there are already many motivations for making precision measurement of the Higgs coupling and testing the new physics beyond the SM.

The Higgs boson discovery is usually regarded as a big success of particle physics, which seems to have completed the SM. Nevertheless, the SM not only requires the existence of Higgs boson, but also dictates its interactions. To fully test the SM, it is necessary to measure all interactions that the Higgs boson participates. Although the spectrum of the SM has already been completed, the SM itself still requires further experimental test. In this sense, the name "particle physics" is somewhat misleading with over-emphasis on its particle content, and we must not forget all the interaction forces between these particles. Even within the SM, we are well motivated to test the properties of the Higgs boson.

The existence of such a $125 \mathrm{GeV}$ scalar is truly profound. It can provide masses to massive gauge bosons and fermions. Its coupling constants with the SM fermions and gauge bosons will be measured by the LHC to about $10 \%-20 \%$ precisions as compared to the SM predictions. ${ }^{4}$ In this sense, we now understand how the mechanism of mass generation can happen with a single vacuum expectation value (VEV). Nevertheless, how the Higgs acquires nonzero VEV has deep connection with the vacuum stability and Higgs inflation, ${ }^{5}$ but has not been tested experimentally yet. ${ }^{6}$ Especially, in the SM the Higgs mass receives quadratic divergence from loop corrections and hence becomes radiatively unnatural if the SM is valid up to very high energy scale. The Higgs mass is radiatively unnatural. In addition, the Yukawa couplings span several orders of magnitude and hence is hierarchically unnatural. A satisfactory model shall make this hierarchical unnaturalness in Yukawa couplings understandable ${ }^{\mathrm{a}}$.

The next-generation lepton colliders, including CEPC, ${ }^{8}$ FCC-ee, ${ }^{9}$ and ILC, ${ }^{10}$ are designed for making precision measurement of the Higgs properties. All three candidate machines can run at $\sqrt{s}=250 \mathrm{GeV}$ as Higgs factory by producing Higgs boson through Higgsstrahlung $e^{+} e^{-} \rightarrow Z h$ and $W W$ fusion $e^{+} e^{-} \rightarrow \nu \bar{\nu} h$ processes. The CEPC with $5 \mathrm{ab}^{-1}$ of integrated luminosity can roughly produce $10^{6}$ Higgs bosons. From a naive estimation, the statistical fluctuation can reach $\mathcal{O}(0.1 \%)$ level for inclusive observables. Considering the fact that the Higgs can decay via various channels, the precision on its coupling can typically reach $\mathcal{O}(1 \%)$ level.

In this talk, we first summarize in Sec. 2 the Higgs coupling precision that

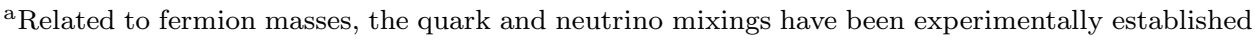
but we are still not sure how to explain. Is the mixing pattern just coincidence or consequence of some flavor symmetry? If there is any flavor symmetry dictating the mixing pattern, what is it? Is the flavor symmetry discrete or continuous? Especially, we shall keep in mind that flavor symmetry has to be broken and the mixing pattern can be determined by residual symmetry that can survive the electroweak symmetry breaking rather than the full flavor symmetry imposed on the fundamental Lagrangian. ${ }^{7}$
} 
can be reached at CEPC based on the assumption that the Higgs production and decay processes can be described by rescaling the SM predictions. We then use the Higgs observables (including both production and decay rates of the Higgs boson), $M_{Z} / M_{W}$ mass measurements, and $Z$-pole observables to estimate the new physics scales via dimension- 6 operators in Sec. 3. Finally, our conclusion can be found in Sec. 4. Interested readers can check our full paper ${ }^{11}$ for details.

\section{Higgs Coupling Precision}

Of the $10^{6}$ Higgs bosons, most of them are produced through the Higgsstrahlung process, $e^{+} e^{-} \rightarrow Z h$. Since there are only two particles in the final state and the initial state is well defined, the Higgs boson can be reconstructed from the $Z$ boson, $p_{h}=-p_{Z}$. This is the so-called recoil mass reconstruction technique ${ }^{12}$ which allows inclusive measurement of the Higgsstrahlung cross section $\sigma(Z h)$. Among the selected events, the Higgs decay rate $\sigma(Z h) \times \operatorname{Br}(h \rightarrow i i)$ of various channels can be measured independently. The ratio of the decay and production rates is the corresponding decay branching ratio $\operatorname{Br}(h \rightarrow i i)$. In this way, the Higgs decay branching ratios can be measured in a model-independent way at lepton colliders. For the $W W$ fusion process, $e^{+} e^{-} \rightarrow \nu \bar{\nu} h$, only the $h \rightarrow b b$ channel has large enough rate $\sigma(\nu \bar{\nu} h) \times \operatorname{Br}(h \rightarrow b b)$. Together with the decay branching ratio $\operatorname{Br}(h \rightarrow b b)$ inferred from Higgsstrahlung, the cross section $\sigma(\nu \bar{\nu} h)$ can also be extracted as the ratio between the directly measured $\sigma(\nu \bar{\nu} h) \times \operatorname{Br}(h \rightarrow b b)$ and the already determined branching ratio $\operatorname{Br}(h \rightarrow b b)$. In Tab. 1, we summarize the direct Higgs observables in black boxes and label the inputs to $\chi^{2}$ fit in red color.

Table 1. The estimated $1 \sigma$ precision of Higgs observables at CEPC. ${ }^{8,13}$ The quantities in black box are experimental observables that can be directly measured while the quantities labeled in red color are the inputs to our $\chi^{2}$ fit analysis.

\begin{tabular}{|c|c|c|c|}
\hline$\Delta \mathrm{M}_{\mathrm{h}}$ & $\Gamma_{\mathbf{h}}$ & $\sigma(\mathbf{Z h})$ & $\boldsymbol{\sigma}(\boldsymbol{\nu} \overline{\boldsymbol{\nu}} \mathbf{h}) \times \operatorname{Br}(h \rightarrow b b)$ \\
\hline \multirow[t]{11}{*}{$2.6 \mathrm{MeV}$} & $2.8 \%$ & $0.5 \%$ & $2.8 \%$ \\
\hline & Decay Mode & $\sigma(Z h) \times \mathrm{Br}$ & $\mathrm{Br}$ \\
\hline & $h \rightarrow b b$ & $0.21 \%$ & $0.54 \%$ \\
\hline & $h \rightarrow c c$ & $2.5 \%$ & $2.5 \%$ \\
\hline & $h \rightarrow g g$ & $1.7 \%$ & $1.8 \%$ \\
\hline & $h \rightarrow \tau \tau$ & $1.2 \%$ & $1.3 \%$ \\
\hline & $h \rightarrow W W$ & $1.4 \%$ & $1.5 \%$ \\
\hline & $h \rightarrow Z Z$ & $4.3 \%$ & $4.3 \%$ \\
\hline & $h \rightarrow \gamma \gamma$ & $9.0 \%$ & $9.0 \%$ \\
\hline & $h \rightarrow \mu \mu$ & $17 \%$ & $17 \%$ \\
\hline & $h \rightarrow$ invisible & - & $0.14 \%$ \\
\hline
\end{tabular}

To extract the precision on the Higgs coupling with the SM particles, we rescale the SM prediction, $g_{h i i} / g_{h i i}^{\mathrm{sm}} \equiv \kappa_{i}$, and parametrize the deviation from the SM as 
$\delta \kappa_{i} \equiv \kappa_{i}-1$. The Higgsstrahlung and $W W$ fusion cross sections are then modulated by the Higgs couplings with $Z$ and $W$ bosons,

$$
\frac{\delta \sigma(Z h)}{\sigma(Z h)}=\kappa_{Z}^{2}-1 \simeq 2 \delta \kappa_{Z}, \quad \frac{\delta \sigma(\nu \bar{\nu} h)}{\sigma(\nu \bar{\nu} h)}=\kappa_{W}^{2}-1 \simeq 2 \delta \kappa_{W},
$$

respectively. Similarly, the decay widths are also modulated by the corresponding rescaling $\kappa$ factors,

$$
\frac{\delta \Gamma_{h i i}}{\Gamma_{h i i}^{\mathrm{sm}}}=\kappa_{i}^{2}-1 \simeq 2 \delta \kappa_{i}, \quad \frac{\Gamma_{i n v}}{\Gamma_{t o t}^{\mathrm{sm}}}=\operatorname{Br}(h \rightarrow \text { invisibles }) \equiv \delta \kappa_{i n v} .
$$

Since no invisible decay mode is present in the SM, its contribution is parametrized directly as $\delta \kappa_{i n v}$ which vanishes when the SM is recovered. In contrast, for those channels already existing in the SM, the deviation $\delta \kappa_{i}$ is the difference from 1 . The total decay width is then the sum over all decay channels, $\Gamma_{\text {tot }}^{\mathrm{sm}} \equiv \sum_{i} \Gamma_{h i i}^{\mathrm{sm}}$.

To fit the quantities labeled as red color in Tab. 1, the rescaled decay widths need to be expressed as decay branching ratios,

$$
\mathrm{Br}_{i}^{\mathrm{th}} \simeq \mathrm{Br}_{i}^{\mathrm{sm}}\left[1+\left(1-\mathrm{Br}_{i}^{\mathrm{sm}}\right) \frac{\delta \Gamma_{i}}{\Gamma_{i}}-\sum_{j \neq i} \mathrm{Br}_{j}^{\mathrm{sm}} \frac{\delta \Gamma_{j}}{\Gamma_{j}}\right],
$$

where $\operatorname{Br}(h \rightarrow i i) \equiv \Gamma_{h i i} / \Gamma_{\text {tot }}$ and $\Gamma_{\text {tot }} \equiv \sum_{i} \Gamma_{h i i}$. Note that the rescaling is no longer an overall factor. For the parametrization of both channels existing in the $\mathrm{SM}$ and the invisible channel in (2), the theoretical prediction can be expanded as,

$$
\mathrm{Br}_{i}^{\mathrm{th}} \simeq \mathrm{Br}_{i}^{\mathrm{sm}}\left(1+\sum_{j} A_{i j} \delta \kappa_{j}\right), \quad \mathrm{Br}_{i n v}^{\mathrm{th}} \simeq \delta \kappa_{i n v},
$$

where the coefficient matrix $A$ has elements,

$$
A_{i j}=2\left(\delta_{i j}-\mathrm{Br}_{j}^{\mathrm{sm}}\right), \quad A_{i, i n v}=-1, \quad A_{i n v, i}=0, \quad A_{i n v, i n v}=1 .
$$

In the decay branching ratios, all decay channels can affect each other. For the channel with larger branching ratio $\mathrm{Br}_{i}^{\mathrm{sm}}$ in the $\mathrm{SM}$, it can have larger effect $\left(A_{j i}=\right.$ $\left.-\mathrm{Br}_{i}^{\mathrm{sm}}\right)$ on the others but smaller effect $\left(A_{i i}=1-\mathrm{Br}_{i}^{\mathrm{sm}}\right)$ on itself. The only exception is the invisible channel which has effect of equal size $\left(A_{i n v, i n v}=1\right.$ for its own and $A_{i, i n v}=-1$ for the others) on all channels. Note that the invisible channel can be affected only by itself.

As summarized in Tab. 1, 9 decay channels from 2 production modes can achieve reasonable precision. To keep the fit as general as possible when estimating the precision on measuring the deviation of Higgs couplings from the SM prediction, 9 scaling $\kappa_{i}(i=b, c, g, \tau, W, Z, \gamma, \mu, i n v)$ are introduced for the nine decay channels, respectively. Note that in the SM, the Higgs decay into a pair of photons or gluons is induced by triangle loops with fermion or $W$ boson that can directly couple to Higgs and hence is not fully independent. Nevertheless, independent scaling factors 


\section{November 21, $2018 \quad$ 4:45 WSPC/INSTRUCTION FILE $\quad$ CEPC`HK}

Testing Higgs Coupling Precision and New Physics Scales at Lepton Colliders 5

Table 2. The $1 \sigma$ precisions on measuring Higgs couplings at CEPC $\left(250 \mathrm{GeV}, 5 \mathrm{ab}^{-1}\right)$, in comparison with LHC $\left(14 \mathrm{TeV}, 300 \mathrm{fb}^{-1}\right)$, HL-LHC $\left(14 \mathrm{TeV}, 3 \mathrm{ab}^{-1}\right)$ and ILC $\left(250 \mathrm{GeV}, 250 \mathrm{fb}^{-1}\right)+\left(500 \mathrm{GeV}, 500 \mathrm{fb}^{-1}\right)$. The numbers for LHC, HL-LHC, and ILC are obtained from. ${ }^{16}$

\begin{tabular}{c|cc|cc|c} 
Precision $(\%)$ & \multicolumn{2}{|c|}{ CEPC } & LHC & HL-LHC & ILC-250+500 \\
\hline$\kappa_{Z}$ & 0.249 & 0.249 & 8.5 & 6.3 & 0.50 \\
$\kappa_{W}$ & 1.21 & 1.21 & 5.4 & 3.3 & 0.46 \\
$\kappa_{\gamma}$ & 4.67 & 4.67 & 9.0 & 6.5 & 8.6 \\
$\kappa_{g}$ & 1.55 & 1.55 & 6.9 & 4.8 & 2.0 \\
$\kappa_{b}$ & 1.28 & 1.28 & 14.9 & 8.5 & 0.97 \\
$\kappa_{c}$ & 1.76 & 1.76 & - & - & 2.6 \\
$\kappa_{\tau}$ & 1.39 & 1.39 & 9.5 & 6.5 & 2.0 \\
$\kappa_{\mu}$ & - & 8.59 & - & - & - \\
$\mathrm{Br}_{\text {inv }}$ & 0.135 & 0.135 & 8.0 & 4.0 & 0.52 \\
$\Gamma_{h}$ & 2.8 & 2.8 & - & - & -
\end{tabular}

$\kappa_{\gamma}$ and $\kappa_{g}$ are assigned to the $h \rightarrow \gamma \gamma$ and $h \rightarrow g g$ decay widths for generality. The $9-$ parameter fit is based on SM and can also accommodate new physics contributions.

Using the technique of analytical $\chi^{2}$ fit $^{11,14}$ delivered in the BSMfitter package ${ }^{15}$ we estimate the precision on Higgs couplings and summarize the results in Tab. 2.

- Roughly speaking, the uncertainty is mainly determined by statistical fluctuations and hence the SM prediction of decay branching ratios $\mathrm{Br}_{i}^{\mathrm{sm}}$.

- Nevertheless, the precision on $\kappa_{Z}$ is much better than the precision on $\kappa_{W}$ although the Higgs decay $h \rightarrow W W\left(\mathrm{Br}_{h \rightarrow W W}^{\mathrm{sm}}=22.5 \%\right)$ has larger branching ratio than $h \rightarrow Z Z\left(\mathrm{Br}_{h \rightarrow Z Z}^{\mathrm{sm}}=2.77 \%\right)$. The additional constraint comes from the Higgsstrahlung cross section $\sigma(Z h)$ which is an inclusive observable and hence has the largest event rate. Similarly, $\kappa_{W}$ also receives constraint from both decay branching ratios and the $W W$ fusion cross section $\sigma(\nu \bar{\nu} h)$.

- Apart from these, the others are only constrained by decay branching ratios. Of all rescaling factors, $\kappa_{\mu}$ has the worst precision since $h \rightarrow \mu \mu\left(\mathrm{Br}_{h \rightarrow \mu \mu}^{\mathrm{sm}}=\right.$ $0.023 \%$ ) has the least number of events and hence the worst statistics. For comparison, we show two fits with or without $\kappa_{\mu}$ and find that all other numbers are not affect. This demonstrates what we argued that a channel $h \rightarrow i i$ can affect other channels with weight $\mathrm{Br}_{i}^{\mathrm{sm}}$. For $h \rightarrow \mu \mu$, its branching ratio $\mathrm{Br}_{h \rightarrow \mu \mu}^{\mathrm{sm}} \simeq 0.023 \%$ is negligibly small.

- Although the branching ratio of $h \rightarrow \gamma \gamma$ is only around $1 \%$ of $h \rightarrow W W$ and hence has 10 times larger uncertainty from naive estimation of statistical fluctuation, the precision on $\kappa_{\gamma}$ is not that worse than $\kappa_{W}$. This is because that photon can be measured much better than the $W$ boson. The photon can be probed directly but the $W$ boson needs to first decay.

- Note that the invisible decay has the best uncertainty as shown in Tab. 1, rendering $\mathrm{Br}_{i n v} \simeq \delta \kappa_{i n v}$ to be mainly determined by this single channel $h \rightarrow$ invisible. We can see that the fitted precision $0.135 \%$ in Tab. 1 is 
roughly the same number as the original value $0.14 \%$ in Tab. 2 since the coefficient $A_{i n v, i n v}$ in (5) is 1 .

- Finally, the combined precision on the total decay width $\Gamma_{h}$ is simply the value directly from detector simulation in Tab. 1 . This is because $\Gamma_{h}$ is an independent variable and not entangled with the rescaling factors $\kappa_{i}$.

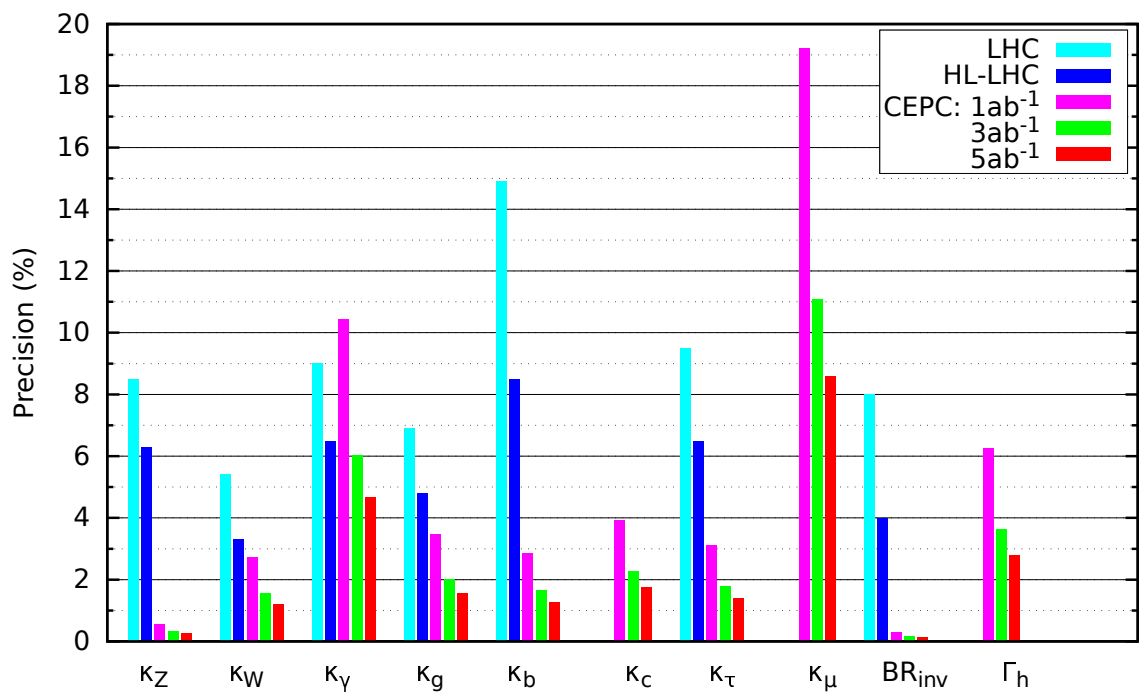

Fig. 1. The $1 \sigma$ precision on the Higgs couplings at CEPC $(250 \mathrm{GeV})$ with integrated luminosity $(1,3,5) \mathrm{ab}^{-1}$, respectively, in comparison with the results at LHC $\left(14 \mathrm{TeV}, 300 \mathrm{fb}^{-1}\right)$ and HL-LHC $\left(14 \mathrm{TeV}, 3 \mathrm{ab}^{-1}\right)$.

For comparison, we also show in Tab. 2 the precision at LHC, HL-LHC, and ILC. ${ }^{16}$ At hadron colliders like LHC and HL-LHC, not all channels can be measured and the precision is usually much worse than at lepton colliders. To make better presentation, the results are depicted in Fig. 1. The reachable precision at CEPC can receive significant improvement from the expected measurement at LHC and HL-LHC. The largest difference appears in $\kappa_{Z}$ and $\mathrm{Br}_{i n v}$ with improvement more than one order of magnitude. In addition, hadron colliders can not measure the Higgs decay width due to large energy uncertainty. For ILC, it can make similar measurements as CEPC. Nevertheless, for most channels the precision is not as good as at CEPC due to smaller integrated luminosity. There are two exceptions, one is $\kappa_{W}$ and the other is $\kappa_{b}$. For $\kappa_{W}$, the enhancement comes from the running at $\sqrt{s}=500 \mathrm{GeV}$ where the $W W$ production of Higgs can be significantly enhanced to introduce better constraint on $\sigma(\nu \bar{\nu} h)=\kappa_{W}^{2} \sigma^{\mathrm{sm}}(\nu \bar{\nu} h)$. 


\section{New Physics Scales}

The purpose of building lepton colliders is not just for precision measurement of the Higgs couplings. Our final goal is new physics beyond the SM. Nevertheless, the energy of lepton colliders is probably not enough to produce the new particles which are usually expected to be heavy. Since the Higgs boson is newly discovered and other SM particles have already been precisely measured to some extent, if there is any new physics, it has large chance to appear in the Higgs couplings. It has large chance for new physics to be measured at lepton colliders indirectly.

The effect of new physics from higher energy can be parametrized in terms of dimension- 6 operators. On the basis of the SM, the Lagrangian is extended by adding effective operators,

$$
\mathcal{L}=\mathcal{L}_{S M}+\sum_{i j} \frac{y_{i j} \sim \mathcal{O}(1)}{\Lambda \sim 10^{14} \mathrm{GeV}}\left(\bar{L}_{i} \widetilde{H}\right)\left(\widetilde{H}^{\dagger} L_{j}\right)+\sum_{i} \frac{c_{i}}{\Lambda^{2}} \mathcal{O}_{i}
$$

where $\widetilde{H} \equiv \tau_{2} H^{*}$ is the CP conjugate of the Higgs doublet $H$. For completeness, we also show the dimension-5 operators which can provide neutrino mass matrix. For $y_{i j} \sim \mathcal{O}(1)$, the tiny neutrino masses can be produced if the cutoff $\Lambda \sim 10^{14} \mathrm{GeV}$ is at the GUT scale. Note that the dimension- 5 operators have lower suppression than the dimension- 6 operators and hence are expected to have larger effect on low-energy physics. This is probably the reason why we have already measured neutrino mass and mixing in neutrino oscillation experiments but have not seen the dimension- 6 operators.

Table 3. The CP-even dimension-6 operators related to Higgs and electroweak precision ob-

\begin{tabular}{|c|c|c|}
\hline Higgs & EW Gauge Bosons & Fermions \\
\hline $\mathcal{O}_{\mathbf{H}}=\frac{1}{2}\left(\partial_{\mu}|\mathbf{H}|^{2}\right)^{2}$ & $\mathcal{O}_{\mathrm{WW}}=g^{2}|\mathbf{H}|^{2} W_{\mu \nu}^{a} W^{a \mu \nu}$ & $\mathcal{O}_{\mathrm{L}}^{(3)}=\left(i \mathbf{H}^{\dagger} \sigma^{a} \stackrel{\leftrightarrow}{D}{ }_{\mu} \mathbf{H}\right)\left(\bar{\Psi}_{L} \gamma^{\mu} \sigma^{a} \Psi_{L}\right)$ \\
\hline $\mathcal{O}_{\mathrm{T}}=\frac{1}{2}\left(\mathbf{H}^{\dagger} \stackrel{\leftrightarrow}{D}_{\mu} \mathbf{H}\right)^{2}$ & $\mathcal{O}_{\mathrm{BB}}=g^{2}|\mathbf{H}|^{2} B_{\mu \nu} B^{\mu \nu}$ & $\mathcal{O}_{L L}^{(3)}=\left(\bar{\Psi}_{L} \gamma_{\mu} \sigma^{a} \Psi_{L}\right)\left(\bar{\Psi}_{L} \gamma^{\mu} \sigma^{a} \Psi_{L}\right)$ \\
\hline & $\mathcal{O}_{\mathrm{WB}}=g g^{\prime} \mathbf{H}^{\dagger} \sigma^{a} \mathbf{H} W_{\mu \nu}^{a} B^{\mu \nu}$ & $\mathcal{O}_{L}=\left(i \mathbf{H}^{\dagger} \stackrel{\leftrightarrow}{D}_{\mu} \mathbf{H}\right)\left(\bar{\Psi}_{L} \gamma^{\mu} \Psi_{L}\right)$ \\
\hline Gluon & $\mathcal{O}_{\mathrm{HW}}=i g\left(D^{\mu} \mathbf{H}\right)^{\dagger} \sigma^{a}\left(D^{\nu} \mathbf{H}\right) W_{\mu \nu}^{a}$ & $\mathcal{O}_{\mathbf{R}}=\left(i \mathbf{H}^{\dagger} \stackrel{\leftrightarrow}{D}_{\mu} \mathbf{H}\right)\left(\bar{\psi}_{R} \gamma^{\mu} \psi_{R}\right)$ \\
\hline $\mathcal{O}_{\mathrm{g}}=g_{s}^{2}|\mathbf{H}|^{2} G_{\mu t}^{a}$ & $\mathcal{O}_{\mathrm{HB}}=i g^{\prime}\left(D^{\mu} \mathbf{H}\right)^{\dagger}\left(D^{\nu} \mathbf{H}\right) B_{\mu \nu}$ & \\
\hline
\end{tabular}

Since the Higgs couplings are the thing to be measured at lepton colliders, we list all related $\mathrm{CP}$-even operators in Tab. 3. All operators involve the Higgs doublet $H$ with the only exception of $\mathcal{O}_{L L}^{(3)}$ which is a four-fermion operator and only affects the Fermi constant $G_{F}$. In this set, all operators are independent by removing redundant operators like $\mathcal{O}_{W}$ and $\mathcal{O}_{B}$. For simplicity, we will not elaborate the details of how these operators can modify the SM prediction of Higgs and electroweak precision observables ${ }^{11}$ and only focus on the physical consequences.

In Tab. 4, we summarize the existing electroweak precision observables ${ }^{17}$ and the Higgs observables at CEPC. ${ }^{8}$ These observables are used to constraint the size 
Table 4. Inputs used to constrain the new physics scales of dimension-6 operators. The electroweak precision observables in the first four rows are taken from PDG2014, ${ }^{17}$ and the $1 \sigma$ precisions of Higgs measurements are taken from the CEPC detector simulations. ${ }^{8}$ For the $W W$ fusion cross section $\sigma[\nu \bar{\nu} h]_{350 \mathrm{GeV}}$ at $\sqrt{s}=350 \mathrm{GeV}$, we adopt the TLEP estimate of its uncertainty ${ }^{9}$ as an illustration.

\begin{tabular}{cccc} 
Observables & Central Value & Relative Error & SM Prediction \\
\hline$\alpha$ & $7.2973525698 \times 10^{-3}$ & $3.29 \times 10^{-10}$ & - \\
$\mathbf{G}_{\mathbf{F}}$ & $1.1663787 \times 10^{-5} \mathrm{GeV}^{-2}$ & $5.14 \times 10^{-7}$ & - \\
$\mathbf{M}_{\mathbf{Z}}$ & $91.1876 \mathrm{GeV}$ & $2.3 \times 10^{-5}$ & - \\
$\mathbf{M}_{\mathbf{W}}$ & $80.385 \mathrm{GeV}$ & $1.87 \times 10^{-4}$ & - \\
\hline$\sigma[Z h]$ & - & $0.51 \%$ & - \\
$\sigma[\nu \bar{\nu} h]$ & - & $2.86 \%$ & - \\
$\sigma[\nu \bar{\nu} h]_{350 \mathrm{GeV}}$ & - & $0.75 \%$ & - \\
\hline $\operatorname{Br}[W W]$ & - & $1.6 \%$ & $22.5 \%$ \\
$\operatorname{Br}[Z Z]$ & - & $4.3 \%$ & $2.77 \%$ \\
$\operatorname{Br}[b b]$ & - & $0.57 \%$ & $58.1 \%$ \\
$\operatorname{Br}[c c]$ & - & $2.3 \%$ & $2.10 \%$ \\
$\operatorname{Br}[g g]$ & - & $1.7 \%$ & $7.40 \%$ \\
$\operatorname{Br}[\tau \tau]$ & - & $1.3 \%$ & $6.64 \%$ \\
$\operatorname{Br}[\gamma \gamma]$ & - & $9.0 \%$ & $0.243 \%$ \\
$\operatorname{Br}[\mu \mu]$ & - & $17 \%$ & $0.023 \%$
\end{tabular}

of dimension- 6 operators with the following $\chi^{2}$ function,

$$
\chi^{2}\left(\delta \alpha, \delta G_{F}, \delta M_{Z}, \frac{c_{i}}{\Lambda^{2}}\right)=\sum_{j}\left[\frac{\mathcal{O}_{j}^{\text {th }}\left(\delta \alpha, \delta G_{F}, \delta M_{Z}, \frac{c_{i}}{\Lambda^{2}}\right)-\mathcal{O}_{j}^{\exp }}{\Delta \mathcal{O}_{j}}\right]^{2},
$$

where $\left(\delta \alpha, \delta G_{F}, \delta M_{Z}\right)$ are shifts of the corresponding parameter $\left(\alpha, G_{F}, M_{Z}\right)$ from their reference values,

$$
\begin{gathered}
\alpha^{(\mathrm{sm})}=\alpha^{(r)}\left(1+\frac{\delta \alpha}{\alpha}\right), \\
G_{F}^{(\mathrm{sm})}=G_{F}^{(r)}\left(1+\frac{\delta G_{F}}{G_{F}}\right), \\
M_{Z}^{(\mathrm{sm})}=M_{Z}^{(r)}\left(1+\frac{\delta M_{Z}}{M_{Z}}\right) .
\end{gathered}
$$

When doing $\chi^{2}$ fit, both the dimension- 6 operator coefficient $c_{j}$ and parameter shifts $\left(\delta \alpha, \delta G_{F}, \delta M_{Z}\right)$ are treated as fitting parameters on the equal footing. For convenience, we take the reference values to be at the experimental central values, $\alpha^{(r)}=7.2973525698 \times 10^{-3}, G_{F}^{(r)}=1.1663787 \times 10^{-5} \mathrm{GeV}^{-2}$, and $M_{Z}^{(r)}=91.1876 \mathrm{GeV}$, while keeping the shifts as small deviations.

This choice of fitting parameters is different from the conventional $Z$-scheme by fixing the input parameters $\left(\alpha, G_{F}, M_{Z}\right)$ to their central values or the $W$-scheme by fixing $\left(\alpha, M_{Z}, M_{W}\right)$ instead. In these scheme-dependent approaches, only the central values of the input parameters are utilized while their uncertainties are simply discarded. The scheme-dependent approach is practically good enough if the input parameters are much more precise than the other observables. This is the case for 
$Z$-scheme with current electroweak precision measurements. As shown in Tab. 4, the relative errors of $\left(\alpha, G_{F}, M_{Z}\right)$ are negligibly small, at least one order of magnitude better than $M_{W}$. Nevertheless, the situation changes when the uncertainty on $M_{W}$ is significantly improved to be comparable with the uncertainty on $M_{Z}$. This is exactly the case for CEPC.

\subsection{Sensitivity Reach from Higgs Observables}

Using all the observables in Tab. 4 (except $\sigma[\nu \bar{\nu} h]_{350 \mathrm{GeV}}$ which is taken from the TLEP estimation) we first estimate the effect of Higgs observables on probing new physics indirectly and show the results in Fig. 2. The 95\% limit (blue) indicates the exclusion sensitivity while the $5 \sigma$ value (red) is the sensitivity for discovery. The following discussions focus on the $95 \%$ limit for simplicity.

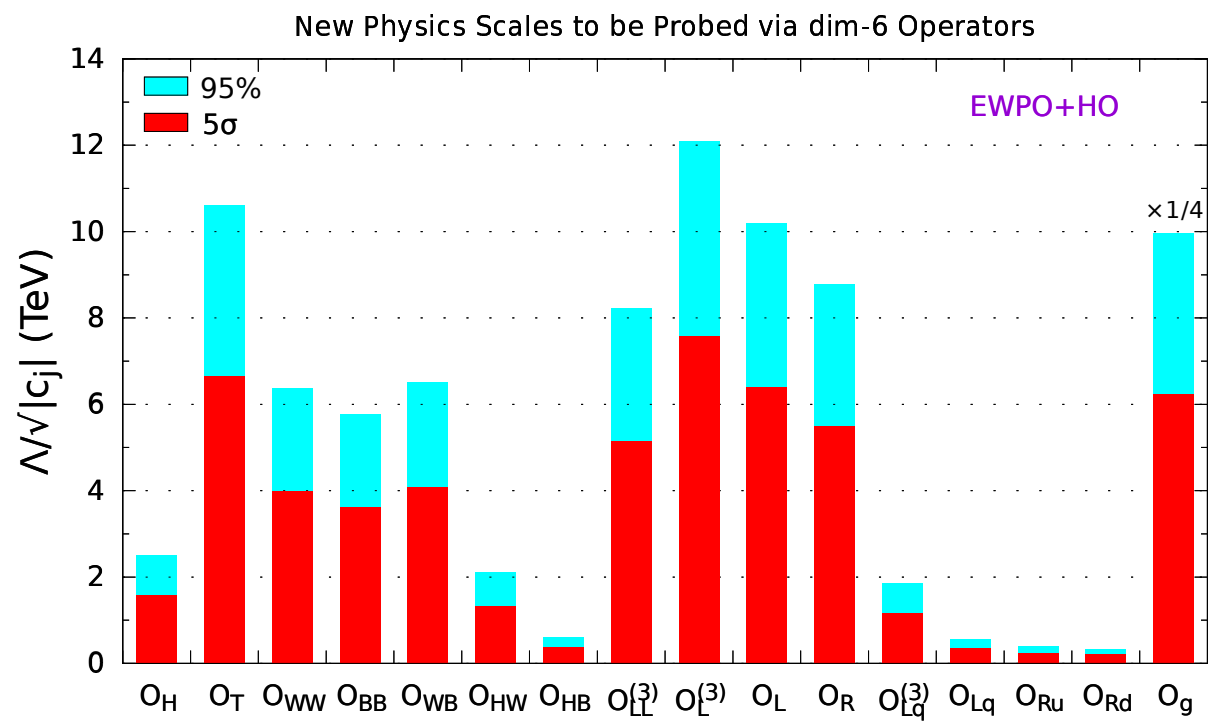

Fig. 2. The $95 \%$ exclusion limits (blue) and $5 \sigma$ discovery sensitivities (red) to the new physics scales $\Lambda / \sqrt{\left|c_{j}\right|}$ by combining the current electroweak precision observables $\left(\alpha, G_{F}, M_{Z}, M_{W}\right)^{17}$ and the future Higgs observables (Table 4$)$ at the Higgs factory CEPC $(250 \mathrm{GeV})^{8}$ with a projected luminosity of $5 \mathrm{ab}^{-1}$. In the last column for $\mathcal{O}_{g}$, we have rescaled its height by a factor $1 / 4$ to fit the plot, so its actual reach is $\Lambda / \sqrt{\left|c_{g}\right|}=39.8 \mathrm{TeV}$.

- We can see that the new physics scales for $\left(\mathcal{O}_{T}, \mathcal{O}_{L}^{(3)}, \mathcal{O}_{L}\right)$ can be probed up to as high as around $10 \mathrm{TeV}$. Half of the operators $\left(\mathcal{O}_{H}, \mathcal{O}_{W W}, \mathcal{O}_{B B}\right.$, $\left.\mathcal{O}_{W B}, \mathcal{O}_{H W}, \mathcal{O}_{L L}^{(3)}, \mathcal{O}_{R}, \mathcal{O}_{L q}^{(3)}\right)$ can be probed up to the scales $2 \sim 10 \mathrm{TeV}$ which is already beyond the effective scale that can be probed at LHC. The remaining $\left(\mathcal{O}_{H B}, \mathcal{O}_{L q}, \mathcal{O}_{R u}, \mathcal{O}_{R d}\right)$ can only be probed below $1 \mathrm{TeV}$.

- Note that the gluon involved operator $\mathcal{O}_{g}$ can even be probed up to $40 \mathrm{TeV}$ already. The reason is that the Higgs decay into a pair of gluons, $h \rightarrow g g$, 
is induced by triangle loops in the SM. In comparison, the contribution of dimension- 6 operators is also at the one-loop level and hence of the same order as the $\mathrm{SM}$ prediction $\mathrm{Br}_{h \rightarrow g g}^{\mathrm{sm}}$. Deviation in this channel then has magnified effect.

\subsection{The Improvement from Electroweak Precision Observables}

Table 5. Impacts of adding the current electroweak precision observables $\left(\alpha, G_{F}, M_{Z}\right.$, $\left.M_{W}\right)^{17}$ on probing the new physics scales $\Lambda / \sqrt{\left|c_{j}\right|}$ (in TeV) at $95 \%$ C.L. The limits in the first row are obtained from $\sigma(Z h)$ to be measured at the $\mathrm{CEPC}^{8}$ only. The limits in the second row are given by combining with the current $M_{W}$ measurement plus $\sigma(Z h)$. Finally, the third row presents the limits by including the current measurements of $\left(\alpha, G_{F}, M_{Z}\right)$ altogether. In the first two rows, $\left(\alpha, G_{F}, M_{Z}\right)$ are fixed to their experimental central values as in the $Z$-scheme, while the third row adopts the scheme-independent approach by allowing all electroweak parameters to freely vary in each fit. We label the entries of most significant improvements in red color with an underscore.

\begin{tabular}{ccccccccccc}
$\mathcal{O}_{H}$ & $\mathcal{O}_{T}$ & $\mathcal{O}_{W W}$ & $\mathcal{O}_{B B}$ & $\mathcal{O}_{W B}$ & $\mathcal{O}_{H W}$ & $\mathcal{O}_{H B}$ & $\mathcal{O}_{L L}^{(3)}$ & $\mathcal{O}_{L}^{(3)}$ & $\mathcal{O}_{L}$ & $\mathcal{O}_{R}$ \\
\hline 2.48 & 2.01 & 4.83 & 0.89 & 1.86 & 2.09 & 0.567 & 5.38 & 11.6 & 10.2 & 8.78 \\
2.48 & $\underline{10.6}$ & 4.83 & 0.89 & $\underline{5.16}$ & 2.09 & 0.567 & $\underline{8.22}$ & 12.1 & 10.2 & 8.78 \\
2.48 & 10.6 & 4.83 & 0.875 & 5.12 & 2.09 & 0.567 & 8.15 & 12.1 & 10.2 & 8.78
\end{tabular}

Among the most sensitive operators, the limit for $\mathcal{O}_{T}$ is mainly provided by $M_{W}$. In Tab. 5 we show the effect of imposing the existing electroweak precision observables. The first row shows the effect of only $\sigma(Z h)$. On this basis, $M_{W}$ is added in the second row and then all of $\left(\alpha, G_{F}, M_{Z}, M_{W}\right)$ in the third row. We can see that the most significant enhancement from $M_{W}$ appears in $\mathcal{O}_{T}$ by promoting its limit from $2 \mathrm{TeV}$ up to $10 \mathrm{TeV}$. In addition, the limits on $\mathcal{O}_{W B}$ and $\mathcal{O}_{L L}^{(3)}$ can also be increased by a factor of $2 \sim 3$. Nevertheless, further imposing the measurements of $\left(\alpha, G_{F}, M_{Z}\right)$ has no effective help since their effect is basically fixing the electroweak parameters $\left(g, g^{\prime}, v\right)$.

\begin{tabular}{|c|c|c|}
\hline Observables & Relative Error & Absolute Error \\
\hline $\begin{array}{l}M_{Z} \\
M_{W}\end{array}$ & $\begin{array}{c}(0.55-1.1) \times 10^{-5} \\
(3.7-6.2) \times 10^{-5}\end{array}$ & $\begin{array}{c}(0.5-1) \mathrm{MeV} \\
(3-5) \mathrm{MeV}\end{array}$ \\
\hline
\end{tabular}

The situation changes when the precision on $M_{W}$ is significantly increased to be comparable with the precision on $M_{Z}$. In Tab. 6 we show the projected $1 \sigma$ precision of $Z$ and $W$ mass measurements at CEPC. ${ }^{8,18}$ Comparing with the existing measurements in Tab. 4, where the precision on $M_{W}$ is almost an order of magnitude worse than the precision on $M_{Z}$, the $M_{W}$ measurement at CEPC is relatively improved more than $M_{Z}$. Fixing $M_{Z}$ to the experimental central value may disguise its interplay with $M_{W}$ as summarized in Tab. 7. On the basis of fitting Higgs and existing electroweak precision observables in Tab. 4 (first row), adding better measurement on $M_{Z}$ (second row) does not improve the reach on new physics scales 
Table 7. Impacts of the projected $M_{Z}$ and $M_{W}$ measurements at $\mathrm{CEPC}^{8,18}$ on the reach of new physics scale $\Lambda / \sqrt{\left|c_{j}\right|}$ (in TeV) at 95\% C.L. The Higgs observables (including $\sigma(\nu \bar{\nu} h$ ) at $350 \mathrm{GeV}$ ) and the existing electroweak precision observables (Table 4) are always included in each row. The differences among the four rows arise from whether taking into account the measurements of $M_{Z}$ and $M_{W}$ (Table 6) or not. The second (third) row contains the measurement of $M_{Z}\left(M_{W}\right)$ alone, while the first (last) row contains none (both) of them. We mark the entries of the most significant improvements from $M_{Z}$ and/or $M_{W}$ measurements in red color with an underscore.

\begin{tabular}{cccccccccccccccc}
$\mathcal{O}_{H}$ & $\mathcal{O}_{T}$ & $\mathcal{O}_{W W}$ & $\mathcal{O}_{B B}$ & $\mathcal{O}_{W B}$ & $\mathcal{O}_{H W}$ & $\mathcal{O}_{H B}$ & $\mathcal{O}_{L L}^{(3)}$ & $\mathcal{O}_{L}^{(3)}$ & $\mathcal{O}_{L}$ & $\mathcal{O}_{R}$ & $\mathcal{O}_{L, q}^{(3)}$ & $\mathcal{O}_{L, q}$ & $\mathcal{O}_{R, u}$ & $\mathcal{O}_{R, d}$ & $\mathcal{O}_{g}$ \\
\hline 2.74 & 10.6 & 6.38 & 5.78 & 6.53 & 2.15 & 0.603 & 8.57 & 12.1 & 10.2 & 8.78 & 1.85 & 0.565 & 0.391 & 0.337 & 39.8 \\
2.74 & $\underline{10.7}$ & 6.38 & 5.78 & $\underline{6.54}$ & 2.15 & 0.603 & $\underline{8.61}$ & 12.1 & 10.2 & 8.78 & 1.85 & 0.565 & 0.391 & 0.337 & 39.8 \\
2.74 & $\underline{21.0}$ & 6.38 & 5.78 & $\underline{10.4}$ & 2.15 & 0.603 & $\underline{\underline{15.5}}$ & $\underline{16.4}$ & 10.2 & 8.78 & 1.85 & 0.565 & 0.391 & 0.337 & 39.8 \\
2.74 & $\underline{23.7}$ & 6.38 & 5.78 & $\underline{11.6}$ & 2.15 & 0.603 & $\underline{17.4}$ & $\underline{18.1}$ & 10.2 & 8.78 & 1.85 & 0.565 & 0.391 & 0.337 & 39.8
\end{tabular}

while the $M_{W}$ measurement at CEPC (third row) can significantly improve the results. The enhancement from $M_{W}$ can be as large as a factor of 2 for $\left(\mathcal{O}_{T}, \mathcal{O}_{W B}\right.$, $\left.\mathcal{O}_{L L}^{(3)}\right)$. It is interesting to see that further imposing the $M_{Z}$ measurement at CEPC, after already imposing $M_{W}$, the sensitivity can be increased by another $10 \%$.

Another feature is the scale of $\mathcal{O}_{H}$ increases from $2.5 \mathrm{TeV}$ in Tab. 5 to $2.74 \mathrm{TeV}$ in Tab. 7 by $10 \%$. This is because of the $W W$ fusion at $\sqrt{s}=350 \mathrm{GeV}$ which has been added into the $\chi^{2}$ fit for Tab. 7. From the Higgsstrahlung peak at $\sqrt{s}=$ $250 \mathrm{GeV}$ to $t \bar{t}$ threshould $\sqrt{s}=350 \mathrm{GeV}$ leads to significant increase in $\sigma(\nu \bar{\nu} h)$ but decrease in $\sigma(Z h)$. Consequently, we can expect the reduced uncertainty in $\sigma(\nu \bar{\nu} h)$, estimated by $\mathrm{TLEP}^{9}$, is the major gain from increasing the lepton collider energy. Nevertheless, the benifit for constraining new physics scales is just $10 \%$.

\subsection{The Improvement from $Z$-Pole Observables}

Table 8. Projected $Z$-pole measurements at $\mathrm{CEPC}^{8,18}$ with integrated luminosity of $5 \mathrm{ab}^{-1}$.

\begin{tabular}{cccccc}
$N_{\nu}$ & $A_{F B}(b)$ & $R^{b}$ & $R^{\mu}$ & $R^{\tau}$ & $\sin ^{2} \theta_{w}$ \\
\hline $1.8 \times 10^{-3}$ & $1.5 \times 10^{-3}$ & $8 \times 10^{-4}$ & $5 \times 10^{-4}$ & $5 \times 10^{-4}$ & $1 \times 10^{-4}$
\end{tabular}

Finally, we further include the $Z$-pole measurements listed in Tab. 8 and show the results in Fig. 3. Now the new physics scales can reach as high as $35 \mathrm{TeV}$, in addition to the $40 \mathrm{TeV}$ of $\mathcal{O}_{g}$. Three operators $\left(\mathcal{O}_{L L}^{(3)}, \mathcal{O}_{L}^{(3)}, \mathcal{O}_{g}\right)$ can be probed up to above $30 \mathrm{TeV}$. Among them, the scales of $\mathcal{O}_{L L}^{(3)}$ and $\mathcal{O}_{L}^{(3)}$ are enhanced by the $Z$-pole observables by almost another factor of 2 . Most of the operators shown in Fig. 3 can be probed above $5 \mathrm{TeV}$, including $\left(\mathcal{O}_{T}, \mathcal{O}_{W W}, \mathcal{O}_{B B}, \mathcal{O}_{W B}, \mathcal{O}_{L}, \mathcal{O}_{R}, \mathcal{O}_{L q}^{(3)}, \mathcal{O}_{L q}\right.$, $\left.\mathcal{O}_{R u}, \mathcal{O}_{R d}\right)$.

Note that the most significant improvement comes from quark related operators $\left(\mathcal{O}_{L q}^{(3)}, \mathcal{O}_{L q}, \mathcal{O}_{R u}, \mathcal{O}_{R d}\right)$. Roughly speaking, the scale of $\mathcal{O}_{L q}^{(3)}$ is increased by a factor of 5 while $\left(\mathcal{O}_{L q}, \mathcal{O}_{R u}, \mathcal{O}_{R d}\right)$ are increased by a factor of 10 . This is because the quark related operators cannot enter the Higgs production cross sections $\sigma(Z h)$ or $\sigma(\nu \bar{\nu} h)$ but can affect the $h \rightarrow Z Z$ and $h \rightarrow W W$ decays indirectly to some extent. Since the Higgs boson mass at $125 \mathrm{GeV}$ is not large enough to put the two $Z / W$ 


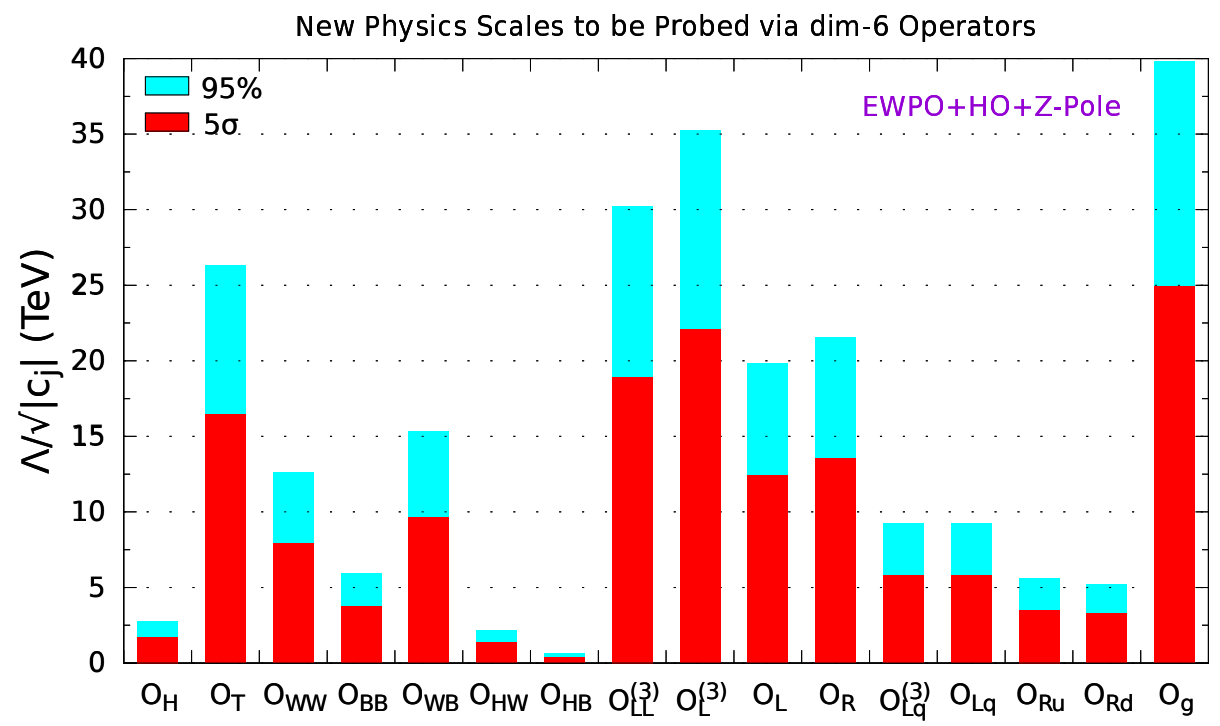

Fig. 3. The $95 \%$ exclusion (blue) and $5 \sigma$ discovery (red) sensitivities to the new physics scales $\Lambda / \sqrt{\left|c_{j}\right|}$ by combining the current electroweak precision measurements $\left(\alpha, G_{F}, M_{Z}, M_{W}\right)^{17}$ with the future Higgs observables at the Higgs factory CEPC (Table 1 ) and $Z$-pole measurements (Table 6) under a projected luminosity of $5 \mathrm{ab}^{-1} .8$

bosons on shell, the decay width has to be calculated with at least one off-shell $Z / W$ boson. Actually, the contribution of two off-shell $Z / W$ can be as large as $25 \%$. Then, the decay width has to be evaluated for the complete chains $h \rightarrow Z Z \rightarrow f_{1} \bar{f}_{i} f_{j} \bar{f}_{j}$ ( $f$ denoting fermions) and $h \rightarrow W W \rightarrow u_{i} \bar{d}_{j} d_{k} \bar{u}_{l}$ ( $u$ for up-type fermion and $d$ for the down-type). The quark related operators then enters as correction to the $Z f \bar{f}$ and $W^{-} u_{i} \bar{d}_{j}$ vertices. Since the $h \rightarrow Z Z$ and $h \rightarrow W W$ channels do not have dominating branching ratios, see Tab. 4 , and the precision on decay branching ratios is not as good as the inclusive measurement of $\sigma(Z h)$, the new physics scales probed by Higgs observables are very low. For $\left(\mathcal{O}_{L, q}, \mathcal{O}_{R, u}, \mathcal{O}_{R, d}\right)$, the sensitivity can only reach $300 \sim 500 \mathrm{GeV}$ while the scale for $\mathcal{O}_{L, q}^{(3)}$ is $1.85 \mathrm{TeV}$. With $Z$-pole measurements, where the $Z f \bar{f}$ and $W^{-} u_{i} \bar{d}_{j}$ vertices dominate, the sensitivities are significantly enhanced to $5 \sim 9 \mathrm{TeV}$.

\section{Conclusion}

The discovery of the Higgs boson at LHC completes the particle spectrum of the SM. Nevertheless, the SM as a whole can be claimed as complete only after fully testing all the interactions that it dictates. The particle physics is not just about particle but also interactions between them. Especially, the scalar Higgs boson mediates new type of interactions. Our current understanding of the Higgs coupling with fermions and Higgs self-interactions is not as good as the gauge interactions. New physics 
may enter by modifying the Higgs coupling with the SM particles. From the point of view of testing the SM or going beyond, a lepton collider (Higgs factory) for precision measurement is necessary.

The next-generation lepton colliders, such as CEPC, FCC-ee, and ILC, are motivated by the discovery of Higgs boson at LHC for precision measurement of its properties. We present the extracted Higgs coupling precision from Higgs observables at CEPC to show its physics potential. With one million events, the Higgs couplings can be measured to $\mathcal{O}(1 \%)$ level. In particular, the Higgs coupling with $Z$ boson can be as good as $0.25 \%$ due to the inclusive cross section of the Higgsstrahlung process $e^{+} e^{-} \rightarrow Z h$.

Although CEPC runs at energy $\sqrt{s}=250 \mathrm{GeV}$ and $Z$-pole, it can probe the new physics beyond the SM indirectly. We use dimension- 6 operators to estimate the new physics scales that can be reached at CEPC. The result shows that the Higgs observables together with existing electroweak precision observables can constrain the new physics up to $10 \mathrm{TeV}$ at $95 \%$ C.L. ( $40 \mathrm{TeV}$ for the gluon related operator $\left.\mathcal{O}_{g}\right)$. If the $M_{Z} / M_{W}$ mass measurements and $Z$-pole observables are also utilized, the new physics scale can be further pushed up to $35 \mathrm{TeV}$. The $Z$-pole observables are as important as the Higgs observables and the $Z$-pole running is useful not only for the purpose of calibration but also for probing the new physics scales. It is beneficial to assign more time for $Z$-pole running at $\sqrt{s} \approx 90 \mathrm{GeV}$ before switching to the Higgs factory mode $\sqrt{s}=250 \mathrm{GeV}$ or returning to $Z$-pole after finishing the Higgs observable measurements. Since CEPC is a circular collider, the $50 \sim 100 \mathrm{~km}$ tunnel can host a hadron $(p p)$ collider SPPC with $\sqrt{s}=50 \sim 100 \mathrm{TeV}$ of energy after CEPC. The energy scale $(10 \sim 40 \mathrm{TeV})$ indirectly reachable at CEPC basically covers the energy range to be effectively explored at SPPC. The lepton collider CEPC can guide and pave the road for the sequent hadron collider SPPC.

Although our study takes CEPC for illustration, the conclusion can apply to other candidate lepton colliders, such as FCC-ee and ILC. The technical details for obtaining the results presented here can be found in our formal paper ${ }^{11}$.

\section{Acknowledgements}

We thank Matthew McCullough, Manqi Ruan, and Tevong You for many valuable discussions. We are grateful to Michael Peskin for discussing the analysis of Ref. ${ }^{16}$ We also thank Timothy Barklow, Tao Han, Zhijun Liang and Matthew Strassler for discussions. SFG is grateful to the Jockey Club Institute for Advanced Study at the Hong Kong University of Science and Technology, especially Henry Tye and Tao Liu, for kind invitation to attend the IAS Program/Conference on High Energy Physics and hospitality during the stay.

\section{References}

1. F. Englert and R. Brout, Phys. Rev. Lett. 13 (1964) 321. P. W. Higgs, Phys. Lett. 12 (1964) 132; Phys. Rev. Lett. 13 (1964) 508; G. S. Guralnik, C. R. Hagen, and T. 
Kibble, Phys. Rev. Lett. 13 (1965) 585; T. Kibble, Phys. Rev. 155 (1967) 1554.

2. G. Aad et al. [ATLAS Collaboration], Phys. Lett. B 716 (2012) 1 [arXiv:1207.7214 [hep-ex]]; S. Chatrchyan et al. [CMS Collaboration], Phys. Lett. B 716 (2012) 30 [arXiv:1207.7235 [hep-ex]].

3. J. Ellis, M. K. Gaillard and D. V. Nanopoulos, "An Updated Historical Profile of the Higgs Boson", [arXiv:1504.07217 [hep-ph]]; and references therein.

4. E.g., J. Ellis and T. You, JHEP 1306 (2013) 103 [arXiv:1303.3879 [hep-ph]]; C. Englert, R. Kogler, H. Schulz, and M. Spannowsky, [arXiv:1511.05170 [hep-ph]].

5. S. F. Ge, H. J. He, J. Ren and Z. Z. Xianyu, Phys. Lett. B 757 (2016) 480 [arXiv:1602.01801]; J. Ellis, H. J. He, and Z. Z. Xianyu, JCAP 1608 (2016) 068 [arXiv:1606.02202]; and Phys. Rev. D 91 (2015) 021302 [arXiv:1411.5537]; H. J. He and Z. Z. Xianyu, JCAP 1410 (2014) 019 [arXiv:1405.7331]; and references therein.

6. E.g., Weiming Yao, [arXiv:1308.6302 [hep-ph]], in the Proceedings of Snowmass Community Summer Study (CSS 2013), July 29-August 6, 2013, Minneapolis, USA; H. J. He, J. Ren, W. Yao, Phys. Rev. D 93 (2015) 015003 [arXiv:1506.03302 [hep-ph]]; A. J. Barr, M. J. Dolan, C. Englert, D. E. Ferreira de Lima, and M. Spannowsky, JHEP 1502 (2015) 016 [arXiv:1412.7154 [hep-ph]]; C. R. Chen and I. Low, Phys. Rev. D 90 (2014) 013018 [arXiv:1405.7040 [hep-ph]]; D. Curtin, P. Meade, C. T. Yu, JHEP 1411 (2014) 127 [arXiv:1409.0005 [hep-ph]]; A. Azatov, R. Contino, G. Panico, and M. Son, Phys. Rev. D 92 (2015) 035001 [arXiv:1502.00539 [hep-ph]]; Qiang Li, Zhao Li, Qi-Shu Yan, Xiaoran Zhao, Phys. Rev. D 92 (2015) 014015 [arXiv:1503.07611 [hep-ph]]; A. V. Kotwal, S. Chekanov and M. Low, Phys. Rev. D 91 (2015) 114018 [arXiv:1504.08042 [hep-ph]]; M. Dall'Osso, T. Dorigo, C. A. Gottardo, A. Oliveira, M. Tosi, and F. Goertz, [arXiv:1507.02245 [hep-ph]]; B. Batell, M. McCullough, D. Stolarski, C. B. Verhaaren, JHEP 1509 (2015) 216 [arXiv:1508.01208 [hep-ph]]; A. Papaefstathiou and K. Sakurai, JHEP 1602 (2016) 006 [arXiv:1508.06524 [hep-ph]]; D. Curtin and P. Saraswat, [arXiv:1509.04284 [hep-ph]]; Chien-Yi Chen, Qi-Shu Yan, Xiaoran Zhao, Zhijie Zhao, Yi-Ming Zhong, Phys. Rev. D 93 (2016) 013007 [arXiv:1510.04013 [hepph]]; Qing-Hong Cao, Y. Liu and B. Yan, [arXiv:1511.03311 [hep-ph]]; R. Grober, M. Muhlleitner, and M. Spira, [arXiv:1602.05851 [hep-ph]]; L. C. Lv, C. Du, Y. Fang, H. J. He, H. Zhang, Phys. Lett. B 755 (2016) 509 [arX iv:1507.02644]; and references therein.

7. S. F. Ge, H. J. He and F. R. Yin, JCAP 1005, 017 (2010) [arXiv:1001.0940 [hepph]]; D. A. Dicus, S. F. Ge and W. W. Repko, Phys. Rev. D 83, 093007 (2011) [arXiv:1012.2571 [hep-ph]]; S. F. Ge, D. A. Dicus and W. W. Repko, Phys. Lett. B 702, 220 (2011) [arXiv:1104.0602 [hep-ph]]; S. F. Ge, D. A. Dicus and W. W. Repko, Phys. Rev. Lett. 108, 041801 (2012) [arXiv:1108.0964 [hep-ph]]; A. D. Hanlon, S. F. Ge and W. W. Repko, Phys. Lett. B 729, 185 (2014) [arXiv:1308.6522 [hep-ph]]; S. F. Ge, [arXiv:1406.1985 [hep-ph]]. H. J. He and F. R. Yin, Phys. Rev. D 84 (2011) 033009 [arXiv:1104.2654]; H. J. He and X. J. Xu, Phys. Rev. D 86 (2012) 111301 (R) [arXiv:1203.2908]; and references therein.

8. CEPC Collaboration, http://cepc.ihep.ac.cn, "CEPC-SPPC Preliminary Conceptual Design Report"; and M. Ruan, [arXiv:1411.5606 [hep-ex]], "Higgs measurement at $e^{+} e^{-}$circular colliders", presentation at 37th International Conference on High Energy Physics (ICHEP-2014), July 2-9, 2014, Valencia, Spain.

9. FCC Collaboration, http://cern.ch/FCC-ee; and M. Bicer et al., JHEP 1401 (2014) 164 [arXiv: 1308.6176 [hep-ex]]; D. d'Enterria, [arXiv:1602.05043 [hep-ex]].

10. H. Baer et al., [arXiv:1306.6352 [hep-ph]]; G. Moortgat-Pick et al., Eur. Phys. J. C 75 (2015) 371 [arXiv:1504.01726 [hep-ph]]; K. Fujii et al., [arXiv:1506.05992 [hep-ex]].

11. S. F. Ge, H. J. He, and R. Q. Xiao, "Probing New Physics Scales from Higgs 
and Electroweak Observables at $e^{+} e^{-}$Higgs Factory," JHEP 1610 (2016) 007 [arXiv:1603.03385].

12. H. Li et al. [ILD Design Study Group Collaboration], "HZ Recoil Mass and Cross Section Analysis in ILD," [arXiv:1202.1439 [hep-ex]].

13. Results presented at "CEPC Physics Software Meeting", IHEP, China, March 26-27, 2016; "CEPC-SPPC Workshop", IHEP, China, April 8-9, 2016.

14. S. F. Ge, K. Hagiwara, N. Okamura and Y. Takaesu, "Determination of mass hierarchy with medium baseline reactor neutrino experiments," JHEP 1305, 131 (2013) [arXiv:1210.8141 [hep-ph]].

15. S. F. Ge, "BSMfitter - Beyond Standard Model Fitter", http://bsmfitter.hepforge.org.

16. M. E. Peskin, "Estimation of LHC and ILC Capabilities for Precision Higgs Boson Coupling Measurements", in the proceedings of Snowmass 2013, [arXiv:1312.4974v3 [hep-ph]].

17. K. A. Olive et al. [Particle Data Group Collaboration], Chin. Phys. C 38 (2014) 090001.

18. H. Yang, H. Li, Q. Li, J. Guo, M. Ruan, Y. Wu, and Z. Liang, presentation of the CEPC Detector Working Group, " $Z$ and $W$ Physics at CEPC",

http://indico.ihep.ac.cn/event/4338/session $/ 2 /$ material $/$ slides $/ 1$ ? contribId $=32$ 